PROCEEDINGS OF THE

AMERICAN MATHEMATICAL SOCIETY

Volume 129, Number 6, Pages 1853-1860

S 0002-9939(00)05740-3

Article electronically published on November 3, 2000

\title{
CONTINUOUS SELECTIONS AND REFLEXIVE BANACH SPACES
}

\author{
VALENTIN GUTEV AND STOYAN NEDEV
}

(Communicated by James E. West)

\begin{abstract}
Every l.s.c. mapping $\Phi$ from a space $X$ into the non-empty closed convex subsets of a reflexive Banach space $Y$ admits a continuous selection provided it satisfies a "weak" u.s.c. condition. This result partially generalizes some known selection theorems. Also, it is successful in solving a problem concerning the set of proper lower semi-continuous convex functions on a reflexive Banach space.
\end{abstract}

\section{INTRODUCTION}

Let $X$ be a topological space, and let $Y$ be a normed space. We let $2^{Y}$ denote the set of all non-empty subsets of $Y$. Also, let

$$
\mathcal{F}(Y)=\left\{S \in 2^{Y}: S \text { is closed }\right\}
$$

and

$$
\mathcal{F}_{c}(Y)=\{S \in \mathcal{F}(Y): S \text { is convex }\} .
$$

A set-valued mapping $\Phi: X \rightarrow 2^{Y}$ is lower semi-continuous, or l.s.c., if the set $\Phi^{-1}(U)=\{x \in X: \Phi(x) \cap U \neq \emptyset\}$ is open in $X$ for every open $U \subset Y$. A map $f: X \rightarrow Y$ is a selection for $\Phi: X \rightarrow 2^{Y}$ if $f(x) \in \Phi(x), x \in X$.

The existence of single-valued continuous selections for l.s.c. mappings $\Phi: X \rightarrow$ $\mathcal{F}_{c}(Y)$ implies some separation properties (like paracompactness, collectionwise normality, etc.) of $X[16]$. A starting point of this paper is the following result of [13] which dispenses with the separation properties of $X$ by strengthening the restriction on the continuity of $\Phi$.

Theorem 1.1. Let $X$ be a topological space, let $Y$ be a Banach space, and let $\Phi$ : $X \rightarrow \mathcal{F}_{c}(Y)$ be d-proximal continuous. Then $\Phi$ admits a single-valued continuous selection.

Let us recall that $\Phi: X \rightarrow \mathcal{F}_{c}(Y)$ is d-proximal continuous if it is l.s.c. and the set $\Phi^{\#}\left(\Phi\left(x_{0}\right)+B_{\varepsilon}\right)=\left\{x \in X: \Phi(x) \subset \Phi\left(x_{0}\right)+B_{\varepsilon}\right\}$ is open in $X$ for every $x_{0} \in X$ and $\varepsilon>0$. Here, $B_{\varepsilon}=\{y \in Y:\|y\|<\varepsilon\}$.

Received by the editors November 18, 1995 and, in revised form, September 27, 1999.

2000 Mathematics Subject Classification. Primary 54C65, 54C60, 46A25; Secondary 54B20, 46B10, 26B25.

Key words and phrases. Set-valued mapping, selection, lower semi-continuous, weakly continuous, hyperspace topology, convex function.

(C)2000 American Mathematical Society 
The main purpose of the paper is to show that, in Theorem 1.1, we can weaken the restriction on the continuity of $\Phi$ provided $Y$ is reflexive. We shall say that $\Phi: X \rightarrow \mathcal{F}_{c}(Y)$ is weakly continuous if it is l.s.c., and the set $\Phi^{\#}(Y \backslash K)$ is open in $X$ for every weakly compact $K \subset Y$. Every $d$-proximal continuous $\Phi: X \rightarrow \mathcal{F}_{c}(Y)$ is weakly continuous, while the converse need not be true (see Propositions 2.5 and 2.6).

Theorem 1.2. Let $X$ be a topological space, let $Y$ be a reflexive Banach space, and let $\Phi: X \rightarrow \mathcal{F}_{c}(Y)$ be weakly continuous. Then $\Phi$ admits a single-valued continuous selection.

Theorem 1.2 works in several interesting situations. So, it applies to l.s.c. mappings $\Phi: X \rightarrow \mathcal{F}_{c}(Y)$ whose Graph is weakly closed (i.e., Graph $(\Phi)=\{(x, y): y \in$ $\Phi(x)\}$ is closed in $X \times Y$, where $Y$ is considered in the weak topology) because every such mapping is weakly continuous (Proposition 2.7). By way of example, the Graph of every upper hemicontinuous mapping is weakly closed (Proposition 2.8). Thus, Theorem [1.2 implies [1, Theorem 9.3.4] when $\Phi$ is l.s.c. and upper hemicontinuous, and $Y$ is a Hilbert space.

Finally, and perhaps most interesting, Theorem 1.2 applies successfully in solving a problem concerning the set $\Gamma(Y)$ of all proper lower semi-continuous convex functions on a reflexive Banach space $Y$. More precisely, if $\operatorname{Aff}(Y)$ denotes the continuous real affine functions on $Y$, then we construct a map $\alpha: \Gamma(Y) \rightarrow \operatorname{Aff}(Y)$ which is continuous with respect to Mosco convergence in $\Gamma(Y)$ and $\operatorname{Aff}(Y)$, and is such that $\alpha(f) \leq f$ (i.e. $\alpha(f)(y) \leq f(y), y \in Y$ ) for every $f \in \Gamma(Y)$ (Theorem 6.1). In case of separable $Y$, this is proved by G. Beer [3] Theorem 3.7].

A proof of Theorem [1.2 is obtained in Section 5. A preparation for that is made in Sections 2, 3 and 4.

\section{WEAKLY CONTINUOUS SET-VALUED MAPPINGS}

Let $X$ be a topological space, let $(Y,\|\|$.$) be a normed space, and let \tau$ be a topology on $\mathcal{F}_{c}(Y)$. We shall say that $\Phi: X \rightarrow \mathcal{F}_{c}(Y)$ is $\tau$-continuous if $\Phi$ is continuous as a mapping from $X$ to the space $\left(\mathcal{F}_{c}(Y), \tau\right)$.

Now, we recall some natural ways for topologizing $\mathcal{F}_{c}(Y)$. The Mosco topology $\tau_{M}$ on $\mathcal{F}_{c}(Y)$ [2], [3], [17, [18] is the one generated by all sets of the form

$$
\langle\mathcal{V}\rangle=\left\{S \in \mathcal{F}_{c}(Y): S \cap V \neq \emptyset, V \in \mathcal{V}, \text { and } S \subset \bigcup \mathcal{V}\right\},
$$

where $\mathcal{V}$ runs over the finite families of open subsets of $Y$ such that $Y \backslash \cup \mathcal{V}$ is weakly compact. The slice topology $\tau_{S}$ on $\mathcal{F}_{c}(Y)$ [5], 6], 20] is the one generated by all sets of the form

$$
\langle\langle\mathcal{V}\rangle\rangle=\left\{S \in\langle\mathcal{V}\rangle: S+B_{\varepsilon} \subset \bigcup \mathcal{V} \text { for some } \varepsilon>0\right\},
$$

where $\mathcal{V}$ is a finite family of open subsets of $Y$ such that $Y \backslash \cup \mathcal{V}$ is a bounded convex subset of $Y$. Finally, we need the so-called Wijsman topology $\tau_{W(d)}$ (see, [12], 15]) generated by all functions

$$
d(y, .): \mathcal{F}(Y) \rightarrow \mathbb{R}, \quad y \in Y,
$$

where $d(y, A)=\inf \{\|y-a\|: a \in A\}, A \in \mathcal{F}(Y)$. Namely, by $\tau_{W(d)}$ we will denote the weakest topology on $\mathcal{F}(Y)$ such that, for every $y \in Y$, the function $d(y,$.$) is$ continuous. 
Our interest in these topologies on $\mathcal{F}_{c}(Y)$ is motivated by the following (usually) strong inclusion:

(2.1) $\tau_{M} \cup \tau_{W(d)} \subset \tau_{S}$.

Indeed, the inclusion $\tau_{W(d)} \subset \tau_{S}$ is obvious. As for $\tau_{M} \subset \tau_{S}$, see [7, Proposition 5.4.11]. On the other hand,

(2.2) $\tau_{M}=\tau_{S}$ if and only if $Y$ is reflexive [7] Corollary 5.4.14].

(2.3) $\tau_{W(d)}=\tau_{M}=\tau_{S}$ if and only if $Y$ is reflexive and the dual norm $\|.\|^{*}$ is weak* Kadec [7, Corollary 5.5.6] (in sequential form [9]).

Proposition 2.4. Let $X$ be a topological space, and let $(Y,\|\|$.$) be a normed space.$ Then $\Phi: X \rightarrow \mathcal{F}_{c}(Y)$ is weakly continuous if and only if it is $\tau_{M}$-continuous.

Proof. This follows from the fact that $\tau_{M}$ has a subbase of the form

$$
\left\{S \in \mathcal{F}_{c}(Y): S \cap V \neq \emptyset\right\} \quad \text { and } \quad\left\{S \in \mathcal{F}_{c}(Y): S \cap K=\emptyset\right\},
$$

where $V$ runs over open and $K$ over weakly compact subsets of $Y$.

Now, we present some examples of weakly continuous set-valued mappings. To this end, we need the proximal topology $\tau_{\delta(d)}$ on $\mathcal{F}(Y)[8$ generated by all sets of the form $\langle\langle\mathcal{V}\rangle\rangle$, where $\mathcal{V}$ is a finite family of open subsets of $Y$. Also, we need the linear topology $\tau_{L}$ on $\mathcal{F}_{c}(Y)$, 4, 14 generated by all sets of the form $\langle\langle\mathcal{V}\rangle\rangle$, where $\mathcal{V}$ is a finite family of open subsets of $Y$ such that $Y \backslash \cup \mathcal{V}$ is convex.

Proposition 2.5. Let $(Y,\|\|$.$) be a normed space. Then every d-proximal contin-$ uous $\Phi: X \rightarrow \mathcal{F}_{c}(Y)$ is weakly continuous.

Proof. Suppose $\Phi: X \rightarrow \mathcal{F}_{c}(Y)$ is $d$-proximal continuous. By [13, Proposition 2.2], $\Phi$ is $\tau_{\delta(d)}$-continuous. Hence, it is $\tau_{S}$-continuous because $\tau_{S} \subset \tau_{\delta(d)}$. Finally, by (2.1) and Proposition 2.4, $\Phi$ is weakly continuous.

Proposition 2.6. Let $Y$ be a separable reflexive Banach space. If every weakly continuous $\Phi: X \rightarrow \mathcal{F}_{c}(Y)$ is d-proximal continuous, then the dimension of $Y$ is at most one.

Proof. Suppose every weakly continuous $\Phi: X \rightarrow \mathcal{F}_{c}(Y)$ is $d$-proximal continuous. Then, by (2.2), Proposition 2.4 and [13, Proposition 2.2], $\Phi: X \rightarrow \mathcal{F}_{c}(Y)$ is $\tau_{\delta(d)^{-}}$ continuous if and only if it is $\tau_{S}$-continuous. That is, $\tau_{\delta(d)}=\tau_{S}$ on $\mathcal{F}_{c}(Y)$. Hence, $\tau_{L}=\tau_{S}$ because $\tau_{S} \subset \tau_{L} \subset \tau_{\delta(d)}$. Now, by [10, 11], $Y$ admits an equivalent norm $\|\cdot\|$ such that $\|.\|^{*}$ is weak ${ }^{*}$ Kadec. In particular, this implies the metrizability of $\left(\mathcal{F}_{c}(Y), \tau_{L}\right)$ because, by $(2.3), \tau_{W(d)}=\tau_{S}=\tau_{L}$ with respect to this norm. Therefore, by [7, Proposition 4.3.10], the dimension of $Y$ is at most one.

Proposition 2.7. Let $X$ be a topological space, let $Y$ be a normed space, and let $\Phi: X \rightarrow \mathcal{F}_{c}(Y)$ be an l.s.c. mapping whose Graph is weakly closed. Then $\Phi$ is weakly continuous.

Proof. Let $K \subset Y$ be weakly compact. Take a point $x_{0} \in \Phi^{\#}(Y \backslash K)$ and $y \in K$. Since $\operatorname{Graph}(\Phi)$ is weakly closed and $\left(x_{0}, y\right) \notin \operatorname{Graph}(\Phi)$, there exists a neighbourhood $U_{y}$ of $x_{0}$ and a weak neighbourhood $V_{y}$ of $y$ such that $U_{y} \times V_{y} \cap \operatorname{Graph}(\Phi)=\emptyset$. In this way, we get a cover $\left\{V_{y}: y \in K\right\}$ of $K$ consisting of weakly open subsets of $Y$. Because of the weak compactness of $K$, there now exists a finite subset $K_{0} \subset K$ such that $K \subset \bigcup\left\{V_{y}: y \in K_{0}\right\}$. Setting then $U_{0}=\bigcap\left\{U_{y}: y \in K_{0}\right\}$, we get a neighbourhood $U_{0}$ of $x_{0}$ such that $\Phi(x) \cap K=\emptyset, x \in U_{0}$. So, $U_{0} \subset \Phi^{\#}(Y \backslash K)$. 
A mapping $\Phi: X \rightarrow \mathcal{F}_{c}(Y)$ is upper hemicontinuous if, for every continuous linear function $p \in Y^{*}$, the function

$$
x \longrightarrow \sigma(\Phi(x), p)=\sup \{p(y): y \in \Phi(x)\} \in \mathbb{R} \cup\{\infty\}, \quad x \in X,
$$

is upper semi-continuous. The following proposition seems to be known (see, for instance, [1, Proposition 2.6.3]).

Proposition 2.8. Let $X$ be a topological space, let $Y$ a Banach space, and let $\Phi: X \rightarrow \mathcal{F}_{c}(Y)$ be upper hemicontinuous. Then, $\operatorname{Graph}(\Phi)$ is weakly closed.

Proof. Since $\Phi$ is closed convex valued, by a version of the Hahn-Banach Separation Theorem,

$$
\Phi(x)=\left\{y \in Y: p(y) \leq \sigma(\Phi(x), p), p \in Y^{*}\right\}, \quad x \in X .
$$

Now, let $\left(x_{0}, y_{0}\right) \notin \operatorname{Graph}(\Phi)$. By $(*)$, there exists $p_{0} \in Y^{*}$ such that $\sigma\left(\Phi\left(x_{0}\right), p_{0}\right)<$ $p_{0}\left(y_{0}\right)-\varepsilon$ for some $\varepsilon>0$. Hence, there exists a neighbourhood $U_{0}$ of $x_{0}$ such that $\sigma\left(\Phi(x), p_{0}\right)<p_{0}\left(y_{0}\right)-\varepsilon$ for every $x \in U_{0}$ because $\sigma\left(\Phi(),. p_{0}\right)$ is upper semicontinuous. Setting then $V_{0}=\left\{y \in Y:\left|p_{0}(y)-p_{0}\left(y_{0}\right)\right|<\varepsilon\right\}$, we get a weak neighbourhood $V_{0}$ of $y_{0}$ such that, by $(*), U_{0} \times V_{0} \cap \operatorname{Graph}(\Phi)=\emptyset$. So, the Graph of $\Phi$ is weakly closed.

\section{A CONTINUity CRITERION In REFleXive BANACH SPACES}

Let $Y$ be a normed space. A norm $\|\cdot\|$ on $Y$ is called locally uniformly rotund if from

$$
\left\|y_{n}\right\|=\|y\|=1 \text { for all } n, \quad \text { and } \lim _{n \rightarrow \infty}\left\|y_{n}+y\right\|=2, y, y_{n} \in Y,
$$

it follows that $\lim _{n \rightarrow \infty}\left\|y_{n}-y\right\|=0$.

Theorem 3.1. Let $(Y,\|\|$.$) be a normed space with \|$.$\| locally uniformly rotund,$ let $X$ be a topological space, $x_{0} \in X$, and let $f: X \rightarrow Y$ be such that the function $\|f()\|:. X \rightarrow \mathbb{R}$ is continuous at $x_{0}$. Also, let $g: X \rightarrow Y$ be a map which is continuous at $x_{0}, g\left(x_{0}\right)=f\left(x_{0}\right)$, and $\|g(x)+f(x)\| \geq 2\|f(x)\|$ for every $x \in X$. Then $f$ is continuous at $x_{0}$.

In what follows, we use $S_{1}$ to denote $\{y \in Y:\|y\|=1\}$. To prove Theorem 3.1 we need the following proposition.

Proposition 3.2. Let $(Y,\|\|$.$) be a normed space with \|$.$\| locally uniformly ro-$ tund, let $X$ be a topological space, $x_{0} \in X$, and let $h: X \rightarrow S_{1}$ be such that $\lim _{x \rightarrow x_{0}}\left\|h(x)+h\left(x_{0}\right)\right\|=2$. Then, $h$ is continuous at $x_{0}$.

Proof. Since $\lim _{x \rightarrow x_{0}}\left\|h(x)+h\left(x_{0}\right)\right\|=2$, for every positive integer $n$ there exists a neighbourhood $U_{n}$ of $x_{0}$ such that

$$
\left|2-\left\|h(x)+h\left(x_{0}\right)\right\|\right|<2^{-n} \quad \text { for every } x \in U_{n} .
$$

Suppose, contrary to what we wish to prove, that $h$ is not continuous at $x_{0}$. Then, there exists an $\varepsilon>0$ such that for every neighbourhood $U$ of $x_{0}$ there is a point $x_{U} \in U$ for which $\left\|h\left(x_{0}\right)-h\left(x_{U}\right)\right\| \geq \varepsilon$. In particular, for every $n$ there exists $x_{n}=x_{U_{n}} \in U_{n}$ such that

$$
\left\|h\left(x_{0}\right)-h\left(x_{n}\right)\right\| \geq \varepsilon .
$$

Now, by (1), $\lim _{n \rightarrow \infty}\left\|h\left(x_{n}\right)+h\left(x_{0}\right)\right\|=2$. Then, by the property of the norm $\|$.$\| ,$ $\lim _{n \rightarrow \infty} h\left(x_{n}\right)=h\left(x_{0}\right)$, which contradicts $(2)$. 
Proof of Theorem [3.1. In the case $\left\|f\left(x_{0}\right)\right\|=0$, there is nothing to prove. So, we may suppose that $\left\|f\left(x_{0}\right)\right\|>0$. Since $\|f()$.$\| is continuous at x_{0}$, there exists a neighbourhood $U_{0}$ of $x_{0}$ such that $\|f(x)\|>0, x \in U_{0}$. Then, define a map $h: X \rightarrow S_{1}$ by

$$
h(x)=\frac{f(x)}{\|f(x)\|} \text { if } x \in U_{0} \quad \text { and } \quad h(x)=h\left(x_{0}\right) \text { otherwise. }
$$

Also, define another map $k: X \rightarrow Y$ by

$$
k(x)=\frac{g(x)}{\|f(x)\|} \text { if } x \in U_{0} \quad \text { and } \quad k(x)=h\left(x_{0}\right) \text { otherwise. }
$$

Now, on the one hand, $\lim _{x \rightarrow x_{0}} k(x)=\frac{f\left(x_{0}\right)}{\left\|f\left(x_{0}\right)\right\|}=h\left(x_{0}\right)$ because $\|f()$.$\| and g$ are continuous at $x_{0}$, and $g\left(x_{0}\right)=f\left(x_{0}\right)$. On the other hand, $x \in U_{0}$ implies

$$
2-\left\|k(x)-h\left(x_{0}\right)\right\| \leq\left\|h(x)+h\left(x_{0}\right)\right\| \leq 2
$$

because

$$
\|k(x)+h(x)\|=\frac{\|g(x)+f(x)\|}{\|f(x)\|} \geq \frac{2\|f(x)\|}{\|f(x)\|}=2\|h(x)\|=2, x \in U_{0} .
$$

So, $\lim _{x \rightarrow x_{0}}\left\|h(x)+h\left(x_{0}\right)\right\|=2$. Hence, by Proposition 3.2 $h$ is continuous at $x_{0}$, and therefore the same is true for $f$ because $f\left|U_{0}=(\|f().\| \cdot h)\right| U_{0}$.

\section{NORM-MINIMAL SELECTIONS}

Let $(Y,\|\|$.$) be a normed linear space. A selection f: X \rightarrow Y$ for $\Phi: X \rightarrow 2^{Y}$ is minimal with respect to the norm, or norm-minimal (see [1]), if

$$
\|f(x)\|=\min \{\|y\|: y \in \Phi(x)\} \quad \text { for every } x \in X .
$$

In what follows, we use $D_{\varepsilon}$ to denote $\{y \in Y:\|y\| \leq \varepsilon\}$.

Theorem 4.1. Let $X$ be a topological space, let $(Y,\|\|$.$) be a reflexive Banach space,$ endowed with a locally uniformly rotund norm $\|$.$\| , and let \Phi: X \rightarrow \mathcal{F}_{c}(Y)$ be l.s.c. The following two conditions are equivalent:

(a) $\Phi^{-1}\left(D_{\varepsilon}\right)$ is closed in $X$ for every $\varepsilon>0$.

(b) $\Phi$ admits a continuous norm-minimal selection.

Proof. Since $\Phi$ is closed convex valued, $Y$ is a reflexive Banach space, and $\|$.$\| is$ locally uniformly rotund, for every $x \in X$ there exists a unique point $f(x) \in \Phi(x)$ such that

$$
\|f(x)\|=\min \{\|y\|: y \in \Phi(x)\} .
$$

In this way, we get a map $f: X \rightarrow Y$ which is a selection for $\Phi$. Since $\Phi$ is l.s.c., the statement of (a) becomes equivalent to continuity of $\|f()$.$\| . Thus, to finish the$ proof, it only remains to show that $f$ is continuous provided $\|f()$.$\| is. To see this,$ we repeat elements of the proof of [19, Theorem]. Namely, take an arbitrary point $x_{0} \in X$. For technical reasons only, let $B_{1 / 0}=Y$. Next, for every integer $n \geq 0$, set

$$
U_{n}=\Phi^{-1}\left(f\left(x_{0}\right)+B_{1 / n}\right) .
$$

Now, for every point $x \in U_{0}=X$, let $n(x)=\sup \left\{n \geq 0: x \in U_{n}\right\}$. Since each $\Phi(x)$ is closed, by (2),

$$
n(x)=\infty \text { implies } f\left(x_{0}\right) \in \Phi(x) .
$$


For every $x \in X$ fix a point $g_{0}(x) \in U_{n(x)} \cap \Phi(x)$, where $U_{\infty}=\left\{f\left(x_{0}\right)\right\}$. In this way, we get a map $g_{0}: X \rightarrow Y$, which is continuous at $x_{0}$. Indeed, $\Phi$ is l.s.c., and therefore, by (2), each $U_{n}, n<\infty$, is open. Then, by (3), this follows from the fact that $g_{0}^{-1}\left(f\left(x_{0}\right)+B_{1 / n}\right)=U_{n}$ for every $n$.

Since $g_{0}$ and $f$ are selections for $\Phi$ and each $\Phi(x)$ is convex, we now get

$$
\frac{g_{0}(x)+f(x)}{2} \in \Phi(x), \quad x \in X .
$$

By (1), this implies $\left\|g_{0}(x)+f(x)\right\| \geq 2\|f(x)\|, \quad x \in X$. Finally, by Theorem 3.1 (with $g=g_{0}$ ), $f$ is continuous at $x_{0}$ because so are $g_{0}$ and $\|f()$.$\| .$

\section{Proof of Theorem 1.2}

Let $A \subset X$. We shall say that a map $g: A \rightarrow Y$ is $A$-regular (see [13]) if for every locally finite cozero-set cover $\mathcal{V}$ of $Y$ there exists a locally finite cozero-set cover $\mathcal{U}$ of $X$ such that $g^{-1}(\mathcal{V})$ is refined by $\mathcal{U} \cap A=\{U \cap A: U \in \mathcal{U}\}$. The following important property of $A$-regularity was actually established in [13].

Proposition 5.1. Let $X$ be a topological space, let $A \subset X, Y$ be a Banach space, and $g: A \rightarrow Y$. Then $g$ can be extended to a continuous map $k: X \rightarrow Y$ if and only if $g$ is A-regular.

In the situation of Theorem 1.2 every weakly continuous mapping admits "sufficiently many" continuous selections. Namely, in this section we establish the following more general selection theorem.

Theorem 5.2. Let $X$ be a topological space, let $Y$ be a reflexive Banach space, and let $\Phi: X \rightarrow \mathcal{F}_{c}(Y)$ be weakly continuous. If $A \subset X$, then every $A$-regular selection $g: A \rightarrow Y$ for $\Phi \mid A$ can be extended to a continuous selection for $\Phi$.

It should be mentioned that Theorem 5.2 partially generalizes [13, Theorem 6.1]. As for its proof, we need the following simple proposition, whose verification is left to the reader.

Proposition 5.3. Let $X$ be a topological space, let $(Y,\|\|$.$) be a normed space, and$ let $\Phi: X \rightarrow \mathcal{F}_{c}(Y)$ be $\tau_{W(d)}$-continuous. Then, for every continuous $h: X \rightarrow Y$, the function $d(h(),. \Phi()):. X \rightarrow \mathbb{R}$ is continuous.

Proof of Theorem 5.2. Let $A \subset X$, and let $g: A \rightarrow Y$ be an $A$-regular selection for $\Phi \mid A$. By Proposition [5.1 $g$ extends to a continuous map $k: X \rightarrow Y$. Next, define another set-valued mapping $\varphi: X \rightarrow \mathcal{F}_{c}(Y)$ by $\varphi(x)=\Phi(x)-k(x), x \in X$. Note that, for every $x \in X$,

$$
0 \in \varphi(x) \quad \text { if and only if } \quad k(x) \in \Phi(x) .
$$

Now, by [21, Theorem 1], $Y$ admits an equivalent norm $\|$.$\| which is locally uni-$ formly rotund. Let us check that the mapping $\varphi$ is $\tau_{W(d)}$-continuous with respect to this norm. Indeed, take a point $y_{0} \in Y$. Then, for every $x \in X$,

$$
d\left(y_{0}, \varphi(x)\right)=\inf \left\{\left\|y_{0}+k(x)-y\right\|: y \in \Phi(x)\right\}=d\left(y_{0}+k(x), \Phi(x)\right) .
$$

Hence, by Proposition 5.3 (with $\left.h(x)=y_{0}+k(x)\right), d\left(y_{0}, \varphi().\right): X \rightarrow \mathbb{R}$ is continuous because, by (2.1), (2.2) and Proposition [2.4, $\Phi$ is $\tau_{W(d)^{-c o n t i n u o u s . ~}}$

Thus, in particular, $\varphi$ is l.s.c. such that $\varphi^{-1}\left(D_{\varepsilon}\right)$ is closed in $X$ for every $\varepsilon>0$. Then, by Theorem 4.1, $\varphi$ admits a continuous minimal selection $\ell: X \rightarrow Y$. Finally, by $(*), f=\ell+k$ is a selection for $\Phi$ which extends $g$. 


\section{LOWER SEMI-CONTINUOUS CONVEX FUNCTIONS AND CONTINUOUS AFFINE FUNCTIONS}

Let $(Y,\|\|$.$) be a linear normed space. The epigraph of a convex function f$ : $Y \rightarrow[-\infty, \infty]$ is the following convex subset of $Y \times \mathbb{R}$ :

$$
\text { epi } f=\{(y, r) \in Y \times \mathbb{R}: r \geq f(y)\} \text {. }
$$

A convex function $f: Y \rightarrow[-\infty, \infty]$ is called proper if its epigraph is non-empty and contains no vertical lines. Let us recall that such a function is lower semicontinuous if and only if its epigraph is closed in $Y \times \mathbb{R}$.

Now, let $\Gamma(Y)$ denote the proper lower semi-continuous convex functions on $Y$, and let $\operatorname{Aff}(Y)$ denote the continuous real affine functions on $Y$. The fundamental notion of convergence for sequences in $\Gamma(Y)$ is Mosco convergence, introduced by U. Mosco [17]: A sequence $\left\{f_{n}\right\} \subset \Gamma(Y)$ is Mosco convergent to $f \in \Gamma(Y)$ if, for every $y \in Y$, (1) there exists a sequence $\left\{y_{n}\right\}$ convergent strongly to $y$ for which $\lim f_{n}\left(y_{n}\right)=f(y)$; and (2) whenever $\left\{y_{n}\right\}$ converges weakly to $y$, then $\liminf f_{n}\left(y_{n}\right) \geq f(y)$.

In the present paper we regard another approach to Mosco convergence in $\Gamma(Y)$. Namely, identifying each function $f \in \Gamma(Y)$ with its epigraph, we may view $\Gamma(Y)$ as a subset of $\mathcal{F}_{c}(Y \times \mathbb{R})$. Then, the Mosco convergence in $\Gamma(Y)$ is compatible with the Mosco topology $\tau_{M}$ in $\mathcal{F}_{c}(Y \times \mathbb{R})[2]$.

In this section, we obtain the following generalization of [3, Theorem 3.7].

Theorem 6.1. Let $Y$ be a reflexive Banach space, $A \subset \Gamma(Y)$, and let $\beta:\left(A, \tau_{M}\right) \rightarrow$ $\left(\operatorname{Aff}(Y), \tau_{M}\right)$ be an $A$-regular map such that $\beta(f) \leq f$ for every $f \in A$. Then, $\beta$ can be extended to a continuous map $\alpha:\left(\Gamma(Y), \tau_{M}\right) \rightarrow\left(\operatorname{Aff}(Y), \tau_{M}\right)$ such that $\alpha(f) \leq f$ for every $f \in \Gamma(Y)$.

Proof. To any $f \in \Gamma(Y)$, we consider its conjugate $f^{*}: Y^{*} \rightarrow[-\infty, \infty]$ defined by the formula:

$$
f^{*}(p)=\sup \{p(y)-f(y): y \in Y\}, \quad p \in Y^{*} .
$$

The so-obtained map $f \rightarrow f^{*}$ is usually called the Young-Fenchel transform and, by [3. Theorem 3.1] (in sequential form [18]), it is a homeomorphism of $\left(\Gamma(Y), \tau_{M}\right)$ onto $\left(\Gamma\left(Y^{*}\right), \tau_{M}\right)$.

In what follows, we also need the map $\psi: Y^{*} \times \mathbb{R} \rightarrow \operatorname{Aff}(Y)$ defined by

$$
\psi(p, r)(y)=p(y)-r, \quad y \in Y .
$$

By [3. Theorem 3.4], this map is a homeomorphism, where $Y^{*}$ is equipped with the norm topology and $\operatorname{Aff}(Y)$ with the Mosco topology.

Now, let $f^{*} \in Y^{*}$, and let us observe that, by (1) and (2),

$$
(p, r) \in \text { epi } f^{*} \quad \text { if and only if } \quad \psi(p, r) \leq f .
$$

Since $Y$ is reflexive, so is the space $Y^{*} \times \mathbb{R}$, where $Y^{*}$ is equipped with the norm topology. Now, let $A^{*}=\left\{f^{*}: f \in A\right\}$, and let $\beta^{*}\left(f^{*}\right)=\psi^{-1}(\beta(f)), f \in A$. Thus, we get an $A^{*}$-regular map $\beta^{*}: A^{*} \rightarrow Y^{*} \times \mathbb{R}$ because $\beta$ is an $A$-regular map and because the Young-Fenchel transform and $\psi$ are homeomorphisms. By $(3), \beta^{*}\left(f^{*}\right) \in$ epi $f^{*}, f^{*} \in A^{*}$. Also, the set-valued mapping: $f^{*} \rightarrow$ epi $f^{*}$ is weakly continuous. Then, by Theorem 5.2 there exists a continuous map $\alpha^{*}$ : $\left(\Gamma\left(Y^{*}\right), \tau_{M}\right) \rightarrow Y^{*} \times \mathbb{R}$ such that $\alpha^{*} \mid A^{*}=\beta^{*}$ and $\alpha^{*}\left(f^{*}\right) \in$ epi $f^{*}, f^{*} \in \Gamma\left(Y^{*}\right)$. Finally, by $(3), \alpha(f)=\psi\left(\alpha^{*}\left(f^{*}\right)\right), f \in \Gamma(Y)$, is as required. 


\section{REFERENCES}

[1] J.-P. Aubin and H. Frankowska, Set-Valued Analysis, System \& Control, Foundation and Applications, vol. 2, Birkhäuser, Boston, 1990. MR 91d:49001

[2] G. Beer, On Mosco convergence of convex sets, Bull. Austral. Math. Soc. 38 (1988), 239-253. MR 90a:46026

[3] - On the Young-Fenchel transform for convex functions, Proc. Amer. Math. Soc. 104 (1988), 1115-1123. MR 89i:49006

[4] - Support and distance functionals for convex sets, Numer. Funct. Anal. Optim. 10 (1989), 15-36. MR 89m:46031

[5] - Topologies on closed convex sets and the Effros measurability of set valued functions, Sém. d'Anal. Convexe Montpellier 21 (1991), exposé No 2. MR 93d:46020

[6] - The slice topology: A viable alternative to Mosco convergence in nonreflexive spaces, Sém. d'Anal. Convexe Montpellier 21 (1991), exposé No 3; Nonlinear Anal. 19 (1992), 271290. MR 93c:52001 MR 93h:49025

[7] _ Topologies on closed and closed convex sets, Mathematics and its applications, vol. 268, Kluwer Academic Publishers, The Netherlands, 1993. MR 95k:49001

[8] G. Beer, A. Lechicki, S. Levi, and S. Naimpally, Distance functionals and suprema of hyperspace topologies, Ann. Mat. Pure Appl. 162 (1992), 367-381. MR 94c:54016

[9] J. Borwein and S. Fitzpatrick, Mosco convergence and the Kadec property, Proc. Amer. Math. Soc. 106 (1989), 843-852. MR 90i:46025

[10] R. Deville, G. Godefroy, and V. Zizler, Smoothness and renormings in Banach spaces, Longman, Harlow, United Kingdom, 1993. MR 94d:46012

[11] J. Diestel, Geometry of Banach spaces, LNM \# 485, Springer-Verlag, Berlin, 1975. MR 57:1079

[12] S. Francaviglia, A. Lechicki, and S. Levi, Quasi-uniformization of hyperspaces and convergence of nets of semicontinuous multifunctions, J. Math. Anal. Appl. 112 (1985), 347-370. MR 87e:54025

[13] V. Gutev, Weak factorizations of continuous set-valued mappings, Topology Appl. 102 (2000), 33-51.

[14] C. Hess, Contributions à l'ètude de la mesurabilité, de la loi de probabilité, et de la convergence des multifunctions, Thèse d'état, Université Montpellier II, 1986.

[15] A. Lechicki and S. Levi, Wijsman convergence in the hyperspace of a metric space, Boll. Un. Mat. Ital. B(7) 1 (1987), 435-452. MR 88e:54007

[16] E. Michael, Continuous selections I, Ann. of Math. 63 (1956), 361-382. MR 17:990e

[17] U. Mosco, Convergence of convex sets and of solutions of variational inequalities, Adv. in Math. 3 (1969), 510-585. MR 45:7560

[18] _ On the continuity of the Young-Fenchel transform, J. Math. Anal. Appl. 35 (1971), 518-535. MR 44:817

[19] S. Nedev, A selection example, C. R. Acad. Bulgare Sci. 40 (1987), no. 11, 13-14. MR 89e:54034

[20] Y. Sonntag and C. Zalinescu, Set convergences: An attempt of classification, Proc. Intr. Conf. on Diff. Equations and Control Theory, Iasi, Romania, August 1990. MR 93a:49008

[21] S. L. Troyanski, On locally uniformly convex and differentiable norms in certain nonseparable Banach spaces, Studia Math. 37 (1971), 173-180. MR 46:5995

School of Mathematical and Statistical Sciences, Faculty of Science, University of Natal, King George V Avenue, Durban 4041, South Africa

E-mail address: gutev@scifs1.und.ac.za

Institute of Mathematics, Bulgarian Academy of Sciences, Acad. G. Bontchev Str., BL. 8, 1113 Sofia, Bulgaria

E-mail address: nedev@math.bas.bg 